\title{
Preoperative Evaluation in Descemet Membrane Endothelial Keratoplasty for Secondary Penetrating Keratoplasty Graft Failure
}

\author{
Khaled Safadi* (D, Ron Kaufman, Eleanor Nche, Denise Wajnsztajn and Itay Lavy \\ Department of Ophthalmology, Hadassah-Hebrew University Medical Center, Jerusalem, Israel

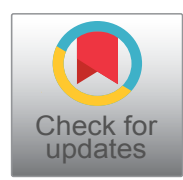

*Corresponding author: Khaled Safadi, MD, Department of Ophthalmology, Hadassah-Hebrew University Medical Center, Jerusalem, Israel

\begin{abstract}
Background: Over the past decade, Penetrating Keratoplasty (PKP) graft failure has been increasingly managed by Descemet Membrane Endothelial Keratoplasty (DMEK). Moreover, Anterior Segment Optical Coherence Tomography (AS-OCT) is becoming an essential modality for perioperative evaluation. Our aim is to share important surgical considerations and modifications based on preoperative evaluation using AS-OCT and, consequently, present our clinical and surgical outcomes of DMEK performed for secondary PKP graft failure.
\end{abstract}

Methods: A retrospective review of medical records was performed for patients who underwent DMEK due to failed PKP in Hadassah Medical Center during 2018-2019. Collected data included demographic characteristics, PKP graft size measured by AS-OCT, corneal donor endothelial cell density (ECD), intra-operative surgical method adjustments, postoperative complications, visual acuity in Snellen (VA), central pachymetry and postoperative ECD.

Results: 16 patients ( 9 males) and 16 eyes were included. The study period was 18 months. Mean age at performing DMEK was 63 years. All patients underwent preoperative AS-OCT and case-based surgical plan was obtained. Before DMEK, mean VA and central pachymetry were 0.04 and $685 \mu \mathrm{m}$, respectively. At last follow up, they significantly improved to $0.3(p$-value $=0.001)$ and $542 \mu \mathrm{m}(\mathrm{p}$-value $=$ 0.008 ) respectively. Postoperatively, $93.75 \%$ of the grafts were attached. Graft failure rate was $6.25 \%$ due to late decompensation. Graft detachment rate and rebubbling rate were $18.75 \%$ respectively.

Conclusion: A suitable case-based preoperative evaluation by AS-OCT may play a vital role in DMEK for failed PKP. Consequently, it utilizes obtaining a suitable surgical plan which may increase success rates and decrease complications rates along with accelerating visual recovery.

\section{Keywords}

DMEK, PKP, Graft failure, Cornea, Anterior segment OCT, Preoperative evaluation

\section{Introduction}

Endothelial keratoplasty (EK) is a viable surgical treatment in patients with secondary graft failure after penetrating keratoplasty (PK) [1]. About a decade ago, secondary PK graft failure was mostly treated by repeating full-thickness PK. However, repeating PKP (rePKP) has multiple complications including a higher risk for allograft rejection, infection from loose sutures and their removal, scarring and thinning of the host cornea from sutures, increased risk for ocular surface disease and slow visual recovery $[2,3]$.

Over the past decade, failed PK graft has been increasingly managed by EK underneath the PK graft in the form of Descemet stripping (automated) endothelial keratoplasty (DSEK/DSAEK) or Descemet membrane endothelial keratoplasty (DMEK) [4-7]. Compared to rePKP, it is believed that EK has a lower risk of allograft rejection and better clinical outcomes $[2,8,9]$. Besides, EK grafts do not require "open-sky" surgery, thereby reducing the risk of intraoperative and postoperative complications [10-14].

In comparison to DSEK/DSAEK, DMEK provides a more selective replacement of the corneal endothelium resulting in smaller incisions, lower risk for allograft

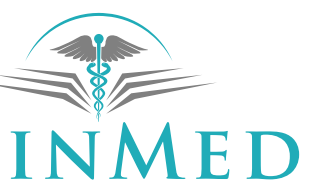

INTERNATIONAL LIBRARY

Citation: Safadi K, Kaufman R, Nche E, Wajnsztajn D, Lavy I (2021) Preoperative Evaluation in Descemet Membrane Endothelial Keratoplasty for Secondary Penetrating Keratoplasty Graft Failure. Int J Ophthalmol Clin Res 8:129. doi.org/10.23937/2378-346X/1410129

Accepted: August 26, 2021: Published: August 28, 2021

Copyright: (c) 2021 Safadi K, et al. This is an open-access article distributed under the terms of the Creative Commons Attribution License, which permits unrestricted use, distribution, and reproduction in any medium, provided the original author and source are credited. 
rejection and better visual and clinical outcomes [15]. Furthermore, the thinner and more flexible DMEK graft may be better suitable for positioning underneath a failed PK graft. It may achieve better apposition than the stiffer DSEK graft, favorable adjustment to the irregular posterior surface or across the posterior PK wound and covers a bigger surface area $[16,17]$. However, the outcomes of DMEK for failed PK graft may not equal those of primary DMEK. Einan-Lifshitz, et al. [18] found higher primary failure rates in DMEK compared with those reported for DSAEK after PKP attributed to persistent postoperative graft detachment. They also found a high long-term failure rate, $43 \%$ of eyes in the DMEK group and $50 \%$ of eyes in the DSAEK group. This may be explained by immune-sensitized eyes due to previous transplants, higher rebubbling rates, intraoperative over-manipulation of the DMEK graft due to compromised anterior chamber structures and a different type of wound-healing response [19].

Our aim is to share important surgical considerations and modifications based on preoperative evaluation using Anterior Segment Optical Coherence Tomography (AS-OCT) and, consequently, present our clinical outcomes of 16 DMEK surgeries performed for secondary PK graft failure.

\section{Materials and Methods}

A retrospective review of data was collected on 16 consecutive eyes of 16 patients who underwent DMEK surgery under a failed PK graft between 2018-2019 at Hadassah Medical Centre in Jerusalem, Israel. The study was conducted in accordance with the tenets of the Declaration of Helsinki and was approved by the ethics committee of the Hebrew University of Jerusalem, Israel.

All surgeries were performed by a single surgeon (I.L) or directly supervised by him. Collected data included demographic characteristics, number of previous corneal transplants, donor corneal endothelial cell density (ECD), intraoperative and postoperative complications including DMEK graft detachment, number of rebubbling procedures, rejection episodes, graft failure, visual acuity in Snellen (VA), central pachymetry and postoperative ECD.

\section{Preoperative graft size assessment and donor tissue preparation}

Each patient underwent Anterior-Segment Optical Coherence Tomography (AS-OCT - Casia 2 Cornea/ Anterior Segment OCT and Accessories, TOMEY GmbH) preoperatively in order to determine the required optimal DMEK graft size according to the original PK graft size and morphological features such as bulging posterior graft-host interface scarring and anterior synechiae, the latter were assessed to consider removing them during surgery (Figure 1 and Figure 2).
From donor globes, corneoscleral buttons were excised less than 24 hours postmortem and stored in organ culture medium at $5{ }^{\circ} \mathrm{C}$. Endothelial cell density was checked using Konan CellChek D Donor Cornea Analytics CD-15 specular microscope. The basic standardized donor tissue preparation technique has already been described [19]. DMEK graft sizes ranged between 7.5-8.5 mm diameters. The required DMEK graft size was assessed by AS-OCT (Figure 1 and Figure 2) according to preoperatively PK graft size.

\section{Surgical technique and multiple adjustments}

The surgical steps for basic standardized DMEK surgical technique has been already described [20]. However, variability in the posterior corneal surface of the recipient due to the presence of the PK graft, as well as the potential restrictions of the PK graft-host junction, required some adjustments and particular manipulations.

Preoperative sub-tenon Triamcinolone $40 \mathrm{mg}$ was injected at the beginning of the surgery after sub-tenon local anaesthesia. The 2.4-mm wide corneal incision was performed in the host peripheral corneal rim without penetrating the PK graft. Descemetorhexis was started from the center of the PK graft and was completed in a curvilinear pattern along the PK wound in a manner resembling capsulorhexis. It was performed under air or fluid maintainer using a reversed Sinskey hook. If scars in the graft-host interface or anterior synechia existed, they were frequently removed with a reversed Sinskey hook, intraocular serrated tweezers, intraocular retinals scissors or a vitrectomy probe accordingly (Figure 3A, Figure 3B and Figure 3C).

The donor Descemet-roll was then injected to the anterior chamber preferably, in a double scroll fashion. After confirming the correct orientation of the graft by intraoperative AS-OCT (Rescan 700 by Zeiss), when available, or the "Moutsouris" sign where the graft is rolled over the tip of the cannula and assures correct orientation of endothelial side down and donor descemet membrane facing the recipient posterior stroma, the graft was unfolded by careful indirect manipulations with air and fluid [20]. In most of the more complicated cases, to prevent over-manipulations of the graft, a small air bubble was injected underneath the donor DM to position the tissue onto the recipient posterior stroma and then centering the graft by the "wave maneuver"; gentle tap and horizontal slide on the top of the cornea with the $27 \mathrm{G}$ cannula tip in a way like the "L" letter (Figure 3D and Figure 3E). Some of the grafts were under-sized, in purpose not to be positioned underneath the PK graft-host junction. In cases with a glaucoma drainage device, the presence of the tube in the anterior chamber required specific additional maneuvers [4].

At the end, $80 \%$ to $100 \%$ of the anterior chamber was 

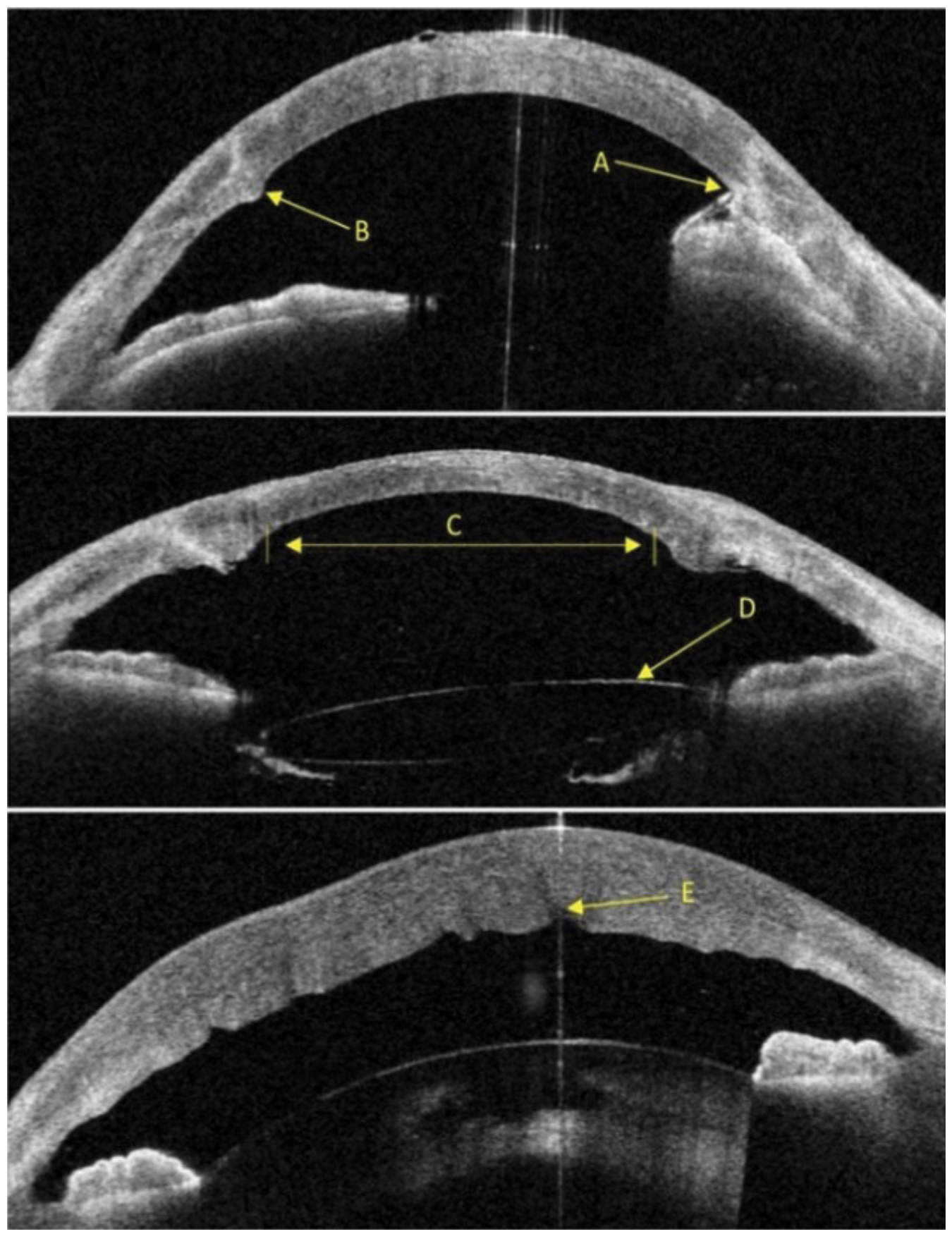

Figure 1: Preoperative Anterior Segment OCT: (A) (arrow) points to anterior synechia adjacent to graft-host interface; (B) (arrow) point to bulging and scarring in the graft-host interface; $(C)$ (arrow) points to the recommended DMEK graft size according to the original PK graft size; (D) (arrow) points to a tilted PCIOL; (E) (arrow) points to deep internal stromal cleft in a severely edematous cornea.

filled with 20\% SF6 gas and the eye was left pressurized (Figure 3F). After 2-3 hours, patients were checked on slit lamp to evaluate the intraocular pressure. If needed, a release of fluid/air/gas from the anterior chamber by pressing on the lower paracentesis with a 27-gauge needle was performed.

\section{Postoperative management}

Postoperative medications included broadspectrum antibiotics for 1 week and steroids; initially dexamethasone 4 times daily for 4 weeks, followed by Fluorometholone 4 times daily. Fluorometholone was gradually tapered to once daily after 9 months.
Postoperative visual acuity was measured using the Snellen visual acuity chart in decimals, postoperative AS-OCT was done to evaluate graft attachment and to measure central pachymetry (Figure 2 ). In case of DMEK graft detachment of more than a third of the graft surface area or central detachment affecting the visual axis, a rebubbling procedure was indicated. The main outcome measures were visual acuity, central pachymetry, rebubbling rate and complications after surgery. Endothelial cell density was checked, when the patient could afford performing the test, using CellChek XL Konan Medical specular microscope. Cystoid macular edema (CME) was assessed one month after surgery using Spectralis Spectral-Domain OCT machine (SD-OCT, 

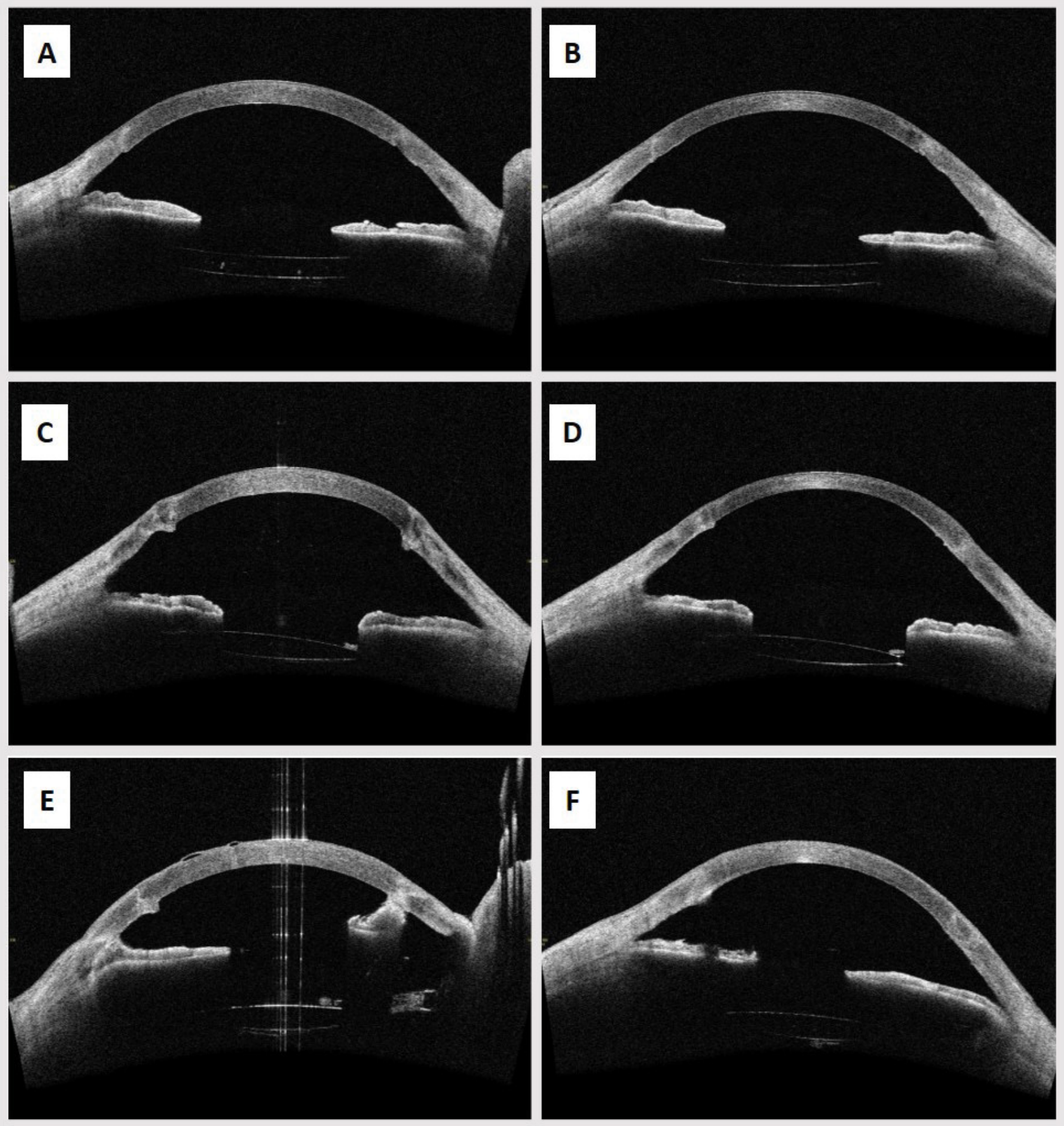

Figure 2: (A) Preoperative AS-OCT showing relatively smooth posterior surface without bulging scars in the PKP-interface, suitable for over-sized graft; (B) Postoperative AS-OCT for the same patient (A) showing fully attached oversized graft; (C) Preoperative AS-OCT showing bulging scars in the PKP interface suggesting the need for removal during surgery and possible transplantation of over-sized graft; (D) Postoperative AS-OCT for the same patient (C) showing fully attached oversized graft after scars removal; (E) Preoperative AS-OCT showing anterior synechia and bulging scars in the PKP interface suggesting the need for additional surgical adjustments and under-sized graft; (F) Postoperative OCT for the same patient (E) showing fully attached under-sized graft after releasing anterior synechia and scars removal.

Heidelberg Engineering, Heidelberg, Germany).

\section{Statistical analysis}

Data were recorded in Microsoft Excel and analyzed using Microsoft Excel and GraphPad Prism 7. For statistical significance testing of normally distributed interval scale parameters, the One-way-ANOVA test was used to compare results preoperatively, one month after surgery and at last follow-up regarding visual acuity and central pachymetry. T-test was used to compare between ECD of the donor and ECD postoperatively. The threshold for statistical significance was defined as P-value $<0.05$.

\section{Results}

\section{Demographic features}

16 patients (9 males, 56.25\%) and 16 eyes were 


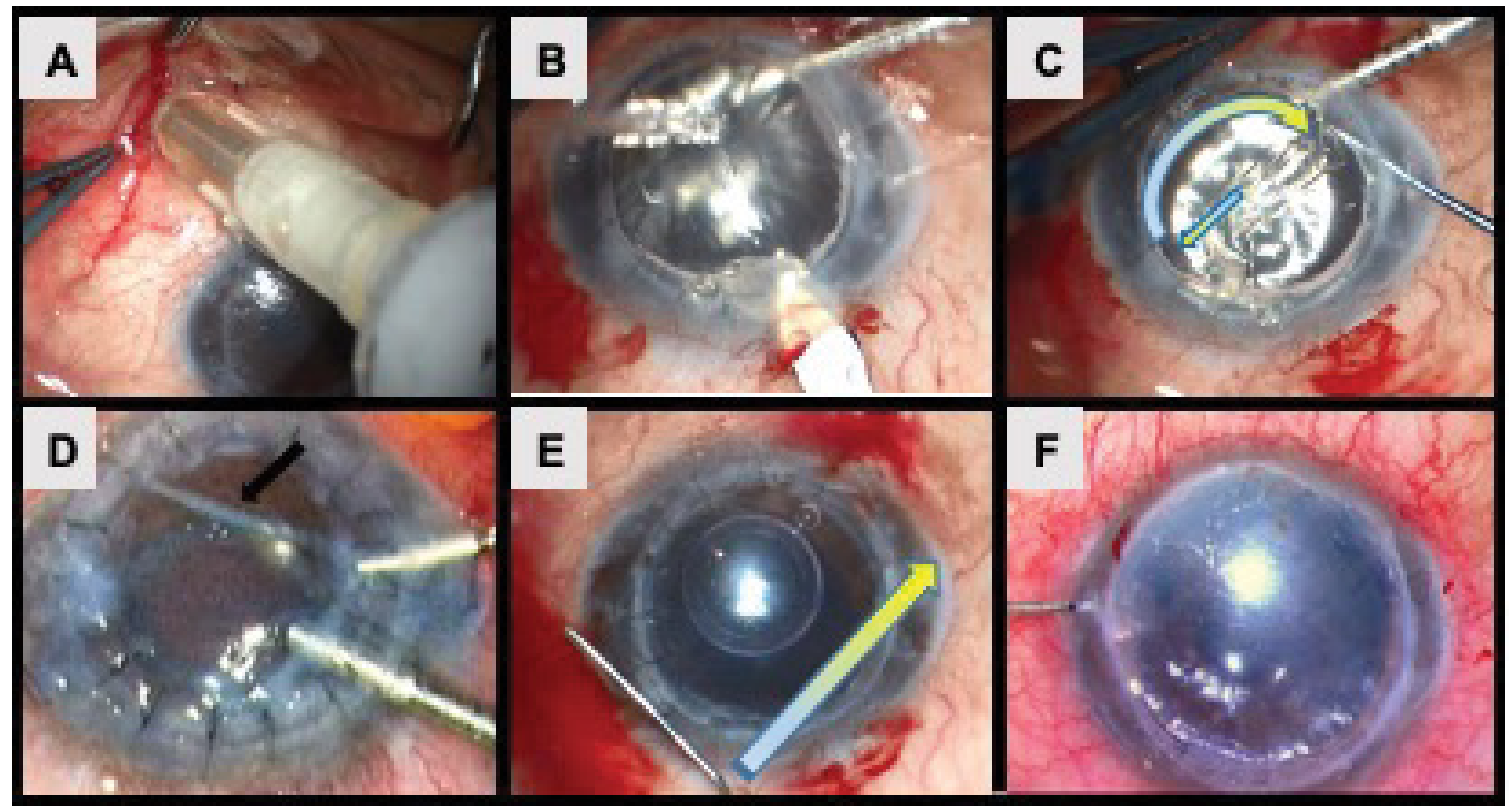

Figure 3: (A) Sub-tenon Triamcinolone $40 \mathrm{mg}$ injected at the beginning of the surgery after sub-tenon local anaesthesia; (B) 2.4-mm wide corneal incision performed in the host peripheral corneal rim; (C) Descemetorhexis performed from the center of the PK graft and completed in a curvilinear pattern along the PK wound; (D) Injected donor descemet-roll to the anterior chamber in a double scroll fashion, and confirmed correct orientation by the "Moutsouris" sign; (E) A small air bubble injected underneath the donor DM and cented graft by "wave maneuver"; $(F)$ At the end of the surgery, the anterior chamber filled $80-100 \%$ with $20 \%$ SF6 gas.

Table 1: Demographic features.

\begin{tabular}{|c|c|}
\hline Patients, Eyes & 16,16 \\
\hline Gender - Male, Female & 9,7 \\
\hline Mean age (range) at DMEK Surgery & 63 years $(37-95)$ \\
\hline \multicolumn{2}{|c|}{ Number of Previous PKP's } \\
\hline One PKP & $10(62.5 \%)$ \\
\hline Two PKP's & $5(31.25 \%)$ \\
\hline Three PKP's & $1(6.25 \%)$ \\
\hline \multicolumn{2}{|c|}{ Initial Indications for PKP } \\
\hline Keratoconus & $11(68.75 \%)$ \\
\hline Bullous Keratopathy & $2(12.5 \%)$ \\
\hline Glaucoma & $1(6.25 \%)$ \\
\hline Trauma & $1(6.25 \%)$ \\
\hline Fungal keratitis & $1(6.25 \%)$ \\
\hline \multicolumn{2}{|c|}{ Lens Status } \\
\hline Phakic & $1(6.25 \%)$ \\
\hline Pseudophakic & $15(93.75 \%)$ \\
\hline \multicolumn{2}{|c|}{ Previous Glaucoma Surgery } \\
\hline Yes & $3(18.75 \%)$ \\
\hline No & $13(81.25 \%)$ \\
\hline
\end{tabular}

included. Mean age \pm SD at performing DMEK was 63 \pm 12.2 years (range 37-95). Before performing DMEK for failed PKP, 10 eyes (62.5\%) underwent one PKP, 5 eyes (31.25\%) underwent two PKP's and 1 eye (6.25\%) underwent three PKP's (Table 1). The study period was 18 months, and hence, the follow up period ranged between 4 to 18 months.

The most common indication for initial PKP was keratoconus in 11 patients (68.75\%). Additional indications included bullous keratopathy in 2 patients $(12.5 \%)$, corneal edema due to glaucoma, trauma and fungal keratitis in one patient each (6.25\%). DMEK surgery was performed for PKP graft failure due to chronic ECD loss. One eye was phakic (6.25\%) and the rest were pseudophakic (93.75\%). Two eyes (12.5\%) had previous glaucoma surgery (Table 1$)$. The PK grafts 
size ranged between 7.75-8.25 $\mathrm{mm}$ in diameter. DMEK grafts were sized according to the principles previously described after preoperative evaluation by AS-OCT for each eye and ranged between 7.5-8.5 $\mathrm{mm}$ in diameter (Figure 1 and Figure 2).

\section{Clinical outcomes}

All surgeries ended with the DMEK graft attached to the posterior part of the PK graft with SF6 bubble supporting the graft. AS-OCT was performed on the same day or a day after the surgery and showed complete attachment of all DMEK grafts.

Mean visual acuity \pm SD before performing DMEK was $0.04 \pm 0.08$, one month after DMEK it increased to $0.16 \pm 0.18$ (P-value $=0.08$ ) and at last follow-up it increased to $0.3 \pm 0.25$ and was statistically significant (P-value $=0.001)$. Regarding mean central pachymetry $\pm \mathrm{SD}$, measured by AS-OCT, before performing DMEK it was $685 \pm 150 \mu \mathrm{m}$, one month after DMEK it decreased to $574 \pm 103.2 \mu \mathrm{m}$ and was statistically significant $(P$-value $=0.04)$ and at last follow-up it decreased to 542 $\pm 100.1 \mu \mathrm{m}$ and was statistically significant $(\mathrm{P}$-value $=$ 0.008) (Table 2).

At last follow-up, 15 out of 16 eyes (93.75\%) had a fully attached graft with a clear cornea (Table 3). Graft failure was observed only in one eye 10 months after surgery (failure rate was $6.25 \%$ ). It was diagnosed with late decompensation and treated successfully with a second DMEK. Three eyes had a partially detached graft during their follow up period and underwent a rebubbling procedure (significant detachment of more than $1 / 3$ of the graft and rebubbling rates were $18.75 \%$ respectively). All rebubbling procedures were successful with fully attached grafts afterward at their last followup; at least 4 months after the procedure. In addition, no rejection episodes were observed along the study follow up period, except in one eye that at last two follow up visits, early rejection signs were suspected. Therefore, immediate anti-inflammatory corticosteroids treatment was administered which successfully hindered the immune reaction and kept the cornea clear.

Regarding corneal endothelial cell density (ECD) (Table 2), donor grafts had a mean ECD \pm SD of $2762 \pm$ 131.2 cells $/ \mathrm{mm}^{2}$. After surgery, on last follow up, only 7 cases had their ECD available with a mean of $1391 \pm$ 902 cells $/ \mathrm{mm}^{2}$. In comparison between the two results, there was a statistically significant difference $(\mathrm{P}$-value $=$ 0.0002).

In order to evaluate postoperative $\mathrm{CME}$, posterior segment SD-OCT for the macula was performed one month after surgery. CME was observed only in one eye out of $16(6.25 \%$, case 12$)$.

\section{Discussion}

Preoperative evaluation is fundamentally important in any medical procedure [21]. We believe that using AS-OCT has a vital role in adjusting the surgical steps for each case individually in DMEK for PK graft failure.

Common conclusions have emerged from multiple previous studies regarding the results of DMEK after PKP compared to primary DMEK $[16,18,19,22,23]$, including higher rebubbling rates, higher endothelial cell density loss, higher late graft failure rates and more demanding surgical technique. Furthermore, glaucoma filtering surgeries, which are probably more prevalent in post-PKP eyes, are considered a significant risk factor for late graft failure, probably as a result of altering the microenvironment in the aqueous humor which accelerates endothelial cell loss [24]. Therefore, based on these eminent conclusions and our experience, additional comprehensive preoperative AS-OCT evaluation was invested to carefully plan DMEK surgery for each case individually, as well as applying multiple adjustments to our surgical technique, in order to decrease failure rates.

Preoperative assessment by AS-OCT aimed to evaluate both the PKP graft size and the presence of

Table 2: Results.

\begin{tabular}{|c|c|c|c|}
\hline & Before surgery & 1-month after surgery & $\begin{array}{c}\text { Last follow-up } \\
(4-18 \text { months })\end{array}$ \\
\hline Mean Visual Acuity (Snellen decimals) & 0.04 & 0.16 (P-value $=0.08)$ & $0.3(\mathrm{P}$-value $=0.001)$ \\
\hline Mean Central Pachymetry $(\mu \mathrm{m})$ & 685 & $574(\mathrm{P}$-value $=0.04)$ & $542(\mathrm{P}$-value $=0.008)$ \\
\hline Mean Endothelial Cells Density $\left(\right.$ cells $\left./ \mathrm{mm}^{2}\right)$ & $2662^{\mathrm{a}}$ & N/A & $1391^{\mathrm{b}}(\mathrm{P}$-value $=0.002)$ \\
\hline
\end{tabular}

a- The mean ECD before surgery in the DMEK donor grafts; b- The mean ECD after DMEK surgery in recipients who performed specular microscopy test.

Table 3: Postoperative complications.

\begin{tabular}{|c|c|}
\hline Attached grafts with clear cornea at last follow-up & $15(93.75 \%)$ \\
\hline Detached grafts after surgery (before rebubbling procedure) & $3(18.75 \%)$ \\
\hline Rebubbling procedures & $3(18.75 \%)$ \\
\hline Graft failure (late decompensation) & $1(6.25 \%)$ \\
\hline Graft rejection & 1 was suspected $(6.25 \%)$ \\
\hline
\end{tabular}


undesired posterior morphological features, including irregular bulging scars in the graft-host interface or anterior synechiae (Figure 1 and Figure 2). Lavy, et al. [19] showed that higher detachment rates occurred in oversized grafts. Posterior bulging of graft-host interface scarring prevents proper graft attachment while anterior synechiae mainly impede intraoperative unfolding DMEK graft and its proper positioning. Therefore, in cases with posterior bulging and no intraoperative plan to dissect the extra tissue, it is important to plan for undersized DMEK graft to reduce the chances of positioning it underneath the PK-host interface and enhance DMEK graft attachment postoperatively (Figure $2 \mathrm{C}$ and Figure 2D). Moreover, evaluating the presence of posterior morphological features and considering their removal during surgery, may decrease intraoperative graft manipulations and facilitate its positioning (Figure 2 , Figure $2 \mathrm{E}$ and Figure $2 \mathrm{~F}$ ). In a smooth, non-bulging posterior surface of the corneal graft host interface, it is worth considering oversizing the DMEK graft, and deliver more endothelial cells to the decompensated cornea (Figure 2A and Figure 2B).

Intra-operative adjustments included injecting sub-tenon triamcinolone prophylactically (Figure $3 \mathrm{~A})$. We believe that it significantly decreases postoperative intraocular inflammation affecting the early postoperative graft failure rate and the incidence of CME. CME is a well-known complication after intraocular surgery and has been reported to occur in $2.0 \%$ to $12.5 \%$ of cases after endothelial keratoplasty [25-27]. Heinzelmann, et al. [25] observed a considerably elevated incidence of CME (13\%) which influences visual rehabilitation. In our study, SD-OCT for macula was performed 1 month after surgery and CME was observed only in one eye (6.25\%), which might be explained by the complicated clinical course of this case. Postoperative intraocular inflammation may also affect graft survival and increase rejection rates which are not negligible after secondary endothelial keratoplasties. Administrating a depo of corticosteroids during surgery may have a beneficial effect on graft survival and endothelial cells function as well.

In addition, paracentesis and main incision were performed in the host peripheral corneal rim without penetrating the PK graft to prevent potential grafthost wound dehiscence (Figure 3B). No circumferential scoring of DM was performed and descemetorhexis was started from the center of the PK graft and completed in a curvilinear pattern along the PK wound in a manner resembling capsulorhexis (Figure $3 \mathrm{C}$ ). We believe, performing these adjustments without removing grafthost interface sutures, may ensue in a smoother back surface, reduce the amount of Descemet remnants and may even facilitate posterior scar tissue removal. Moreover, scars in the graft-host interface or anterior synechia, were selectively removed during surgery, if possible, based upon preoperative AS-OCT (Figure 3C).
After injecting the DMEK graft, the correct orientation was confirmed by intraoperative AS-OCT (Rescan), when available, or the "Moutsouris" sign. Afterward, the graft was unfolded using careful indirect manipulations by tapping on the cornea surface. Then, the graft was elevated with a small air bubble beneath and was centered by the "wave maneuver", an indirect $L$ shaped tapping on the corneal surface (Figure $3 D$ and Figure $3 \mathrm{E}$ ). We believe that these surgical steps lessen unnecessary extra manipulations on the graft and facilitate positioning it in the correct orientation and suitable position. This way of manipulating the graft on the posterior corneal surface with Descemet-stromal touch, helps to avoid endothelial-iris/IOL touch, which may also protect the graft endothelial cells.

Finally, $80 \%$ to $100 \%$ of the anterior chamber was filled with $20 \%$ SF6 gas to pressurize the eye and support graft adherence for a longer period than air (Figure 3F). After Two to three hours, intraocular pressure was checked and if necessary, an appropriate intervention was applied. Lavy, et al. [19] attributed the tendency of delayed and extensive graft detachment, to insufficient pressurization during surgery or postoperative hypotonia. They recommended to pay attention for pressurizing the eye at the end of surgery or to extend the air-bubble time if enough pressurization cannot be achieved. Compared to other studies, we had relatively low rebubbling rate $(18.75 \%)$, this may be attributed to SF6 usage and the extended volume in the anterior chamber at the end of the surgery, but also may be due to a more conservative rebubbling policy of the surgeon.

In our study, we encountered relatively low complications rates. Graft failure rate was $6.25 \%$ (late), graft detachment rate and rebubbling rate were $18.75 \%$ respectively. Interestingly, glaucoma filtering surgeries were not a significant risk factor for graft failure. However, this conclusion may be applicable at least regarding early decompensation events and not for late decompensation events due to short-term study period. We believe, based on our experience, that these successful results are attributed to our careful preoperative AS-OCT evaluation and intra-operative adjustments to the surgical technique of DMEK after PKP graft failure. In comparison to our results, higher rebubbling and failure rates were reported by other studies. Lifshitz, et al. [18] had $43 \%$ rebubbling and $43 \%$ failure rates. Lavy, et al. [19] found $34 \%$ and $36 \%$ rebubbling and failure rates respectively. Heinzelmann, et al. [23] reported $37 \%$ and $21 \%$ rebubbling and failure rates respectively.

Visual recovery and central pachymetry improvement were relatively fast during the study period (Table 2). Those favorable clinical outcomes are consistent with other studies as well $[16,18,22]$. The visual recovery with DMEK under failed PK contrasts sharply with the delayed and unpredictable visual rehabilitation after 
re-PKP [28]. Moreover, the rapid visual recovery seen after DMEK under failed PK is consistent with the visual recovery that DMEK provides in virgin eyes as compared with PK [29].

There are some limitations to our study, including its retrospective nature, small sample size and shortterm follow up. Moreover, visual acuity tests were done by technicians and not experienced optometrists. Therefore, uncorrected or partially corrected, rather than best corrected, visual acuity was assessed. In addition, ECD was done only for 7 cases after surgery and comparison between donors ECD and postoperative ECD may be inconclusive due to group size difference. This was mainly because specular microscopy test was not covered by the health insurance and not all study participants could afford it. However, we encountered a predictable decrease in ECD after surgery which may be attributed to cell migration and postoperative inflammatory response [19].

\section{Conclusions}

DMEK is a viable method to treat secondary PKP graft failure. It may provide faster, and better visual recovery and clinical outcomes compared to re-PKP. Preoperative evaluation using AS-OCT, where available, plays a key role in planning the surgery based on each case characteristic. No less important is adhering to surgical adjustments during DMEK surgery. Both may further increase success rates and decrease the incidence of graft detachment, rebubbling and failure rates.

\section{Data Availability}

Data will not be shared because the authors are performing other analyses that have not yet been published.

\section{Conflicts of Interest}

The authors declare that they have no conflict of interest.

\section{Funding Statement}

This study received no specific grant from any funding agency in the public, commercial or not-forprofit sectors.

\section{Acknowledgments}

The manuscript has been submitted as a Preprint in Research Square (DOI: 10.21203/rs.3.rs-34409/v1).

\section{References}

1. Straiko MD, Terry MA, Shamie N (2011) Descemet stripping automated endothelial keratoplasty under failed penetrating keratoplasty: A surgical strategy to minimize complications. Am J Ophthalmol 151: 233-7.e2.

2. Claesson M, Armitage WJ (2013) Clinical outcome of repeat penetrating keratoplasty. Cornea 32: 1026-1030.

3. Lee WB, Shtein RM, Kaufman SC, Deng SX, Rosenblatt
MI, et al. (2015) Boston Keratoprosthesis: Outcomes and Complications: A Report by the American Academy of Ophthalmology. Ophthalmology 122: 1504-1511.

4. Liarakos VS, Satué M, Livny E, Van Dijk K, Ham L, et al. (2015) Descemet membrane endothelial keratoplasty for a decompensated penetrating keratoplasty graft in the presence of a long glaucoma tube. Cornea 34: 1613-1616.

5. Ang $\mathrm{M}$, Ho $\mathrm{H}$, Wong $\mathrm{C}$, Htoon HM, Mehta JS, et al. (2014) Endothelial keratoplasty after failed penetrating keratoplasty: An alternative to repeat penetrating keratoplasty. Am J Ophthalmol 158: 1221-1227.e1.

6. Price FW, Price MO (2006) Endothelial keratoplasty to restore clarity to a failed penetrating graft. Cornea 25: 895899.

7. Covert DJ, Koenig SB (2007) Descemet stripping and automated endothelial keratoplasty (DSAEK) in eyes with failed penetrating keratoplasty. Cornea 26: 692-696.

8. Sellami D, Abid S, Bouaouaja G, Ben Amor S, Kammoun B, et al. (2007) Epidemiology and Risk Factors for Corneal Graft Rejection. Transplant Proc 39: 2609-2611.

9. Panda A, Vanathi M, Kumar A, Dash Y, Priya S (2007) Corneal Graft Rejection. Surv Ophthalmol 52: 375-396.

10. Einan-Lifshitz A, Sorkin N, Boutin T, Showail M, Borovik A, et al. (2017) Comparison of femtosecond laser-enabled descemetorhexis and manual descemetorhexis in descemet membrane endothelial keratoplasty. Cornea 36: 767-770.

11. Borovik AM, Perez M, Lifshitz T, Einan-Lifshitz A, Sorkin N, et al. (2017) Peripheral Blunt Dissection: Using a MicrohoeFacilitated Method for Descemet Membrane Endothelial Keratoplasty Donor Tissue Preparation. Cornea 36: 12701273.

12. Guerra FP, Anshu A, Price MO, Giebel AW, Price FW (2011) Descemet's membrane endothelial keratoplasty: Prospective study of 1-year visual outcomes, graft survival, and endothelial cell loss. Ophthalmology 118: 2368-2373.

13. Melles GRJ, Ong TS, Ververs B, van der Wees J (2006) Descemet Membrane Endothelial Keratoplasty (DMEK). Cornea 25: 987-990.

14. Yoeruek E, Bayyoud T, Hofmann J, Bartz-Schmidt KU (2013) Novel maneuver facilitating Descemet membrane unfolding in the anterior chamber. Cornea 32: 370-373.

15. Anshu A, Price MO, Tan DTH, Price FW (2012) Endothelial Keratoplasty: A Revolution in Evolution. Surv Ophthalmol 57: 236-252.

16. Anshu A, Price MO, Price FW (2013) Descemet membrane endothelial keratoplasty and hybrid techniques for managing failed penetrating grafts. Cornea 32: 1-4.

17. Keane MC, Galettis RA, Mills RAD, Coster DJ, Williams KA (2016) A comparison of endothelial and penetrating keratoplasty outcomes following failed penetrating keratoplasty: A registry study. Br J Ophthalmol 100: 15691575.

18. Einan-Lifshitz A, Belkin A, Sorkin N, Mednick Z, Boutin T, et al. (2020) Descemet membrane endothelial keratoplasty after penetrating keratoplasty: Features for success. Cornea 37: 1093-1097.

19. Lavy I, Liarakos VS, Verdijk RM, Parker J, Müller TM, et al. (2017) Outcome and histopathology of secondary penetrating keratoplasty graft failure managed by descemet membrane endothelial keratoplasty. Cornea 36: 777-784.

20. Dapena I, Moutsouris K, Droutsas K, Ham L, Van Dijk K, et 
al. (2011) Standardized "no-touch" technique for descemet membrane endothelial keratoplasty. Arch Ophthalmol 129: 88-94.

21. O'Donnell FT (2016) Preoperative Evaluation of the Surgical Patient. Mo Med 113: 196-201.

22. Pasari A, Price MO, Feng MT, Price FW (2019) Descemet Membrane Endothelial Keratoplasty for Failed Penetrating Keratoplasty: Visual Outcomes and Graft Survival. Cornea 38: 151-156.

23. Heinzelmann S, Böhringer $D$, Eberwein $P$, Lapp $T$, Reinhard T, et al. (2017) Descemet membrane endothelial keratoplasty for graft failure following penetrating keratoplasty. Graefe's Arch Clin Exp Ophthalmol 255: 979985.

24. Rosenfeld C, Price MO, Lai X, Witzmann FA, Price FW (2015) Distinctive and pervasive alterations in aqueous humor protein composition following different types of glaucoma surgery. Mol Vis 21: 911-918.

25. Heinzelmann S, Maier P, Böhringer D, Hüther S, Eberwein $P$, et al. (2015) Cystoid macular oedema following Descemet membrane endothelial keratoplasty. $\mathrm{Br} \mathrm{J}$ Ophthalmol 99: 98-102.

26. Phillips PM, Phillips LJ, Much JW, Maloney C (2012) Descemet stripping endothelial keratoplasty: Six-month results of the first 100 consecutive surgeries performed solo by a surgeon using 1 technique with $100 \%$ follow-up. Cornea 31: 1361-1364.

27. Suh LH, Yoo SH, Deobhakta A, Donaldson KE, Alfonso EC, et al. (2008) Complications of Descemet's Stripping with Automated Endothelial Keratoplasty. Survey of 118 Eyes at One Institute. Ophthalmology 115: 1517-1524.

28. Al-Mezaine H, Wagoner MD (2006) Repeat penetrating keratoplasty: Indications, graft survival, and visual outcome $\mathrm{Br} J$ Ophthalmol 90: 324-327.

29. Deng SX, Lee WB, Hammersmith KM, Kuo AN, Li JY, et al. (2018) Descemet Membrane Endothelial Keratoplasty: Safety and Outcomes: A Report by the American Academy of Ophthalmology. Ophthalmology 125: 295-310. 